\title{
Robert Kellner Lecture awarded to ABC author Bernhard Lendl
}

\author{
Nicola Oberbeckmann-Winter ${ }^{1}$
}

Received: 23 March 2016/Accepted: 24 March 2016/Published online: 21 April 2016

(C) Springer-Verlag Berlin Heidelberg 2016

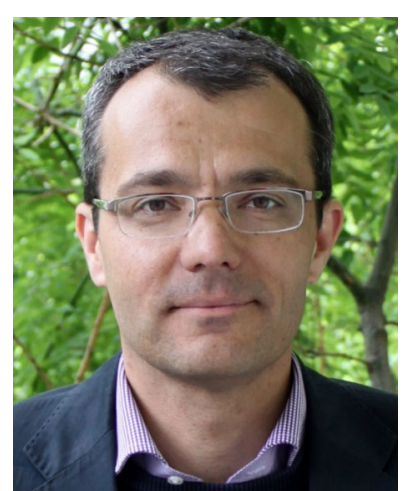

Bernhard Lendl received his $\mathrm{PhD}$ in chemistry from Technische Universität Wien (TU Wien) in 1996. He was appointed associate professor of analytical chemistry in 2001 at the same university, and from 2003 to 2004 he was guest professor at the University of Córdoba, Spain. In 2008 he co-founded QuantaRed Technologies, where he acts as scientific director. Since 2011 he has been the head of the Division of Environmental and Process Analytical Chemistry at TU Wien. In 2016 he was appointed full professor of vibrational spectroscopy at TU Wien. His research focuses on the development of novel methods and technologies based on infrared and Raman spectroscopies for use in analytical chemistry. His current work centers on mid-IR quantum cascade lasers for environmental and process monitoring, lab-on-a-chip systems for timeresolved mid-IR spectroscopy, ultrasound-enhanced mid-IR fiber-optic sensors, surface-enhanced Raman scattering, and stand-off Raman spectroscopy. His work is summarized in more than 200 reviewed papers and has led to six international patent applications.

Nicola Oberbeckmann-Winter

nicola.oberbeckmann-winter@springer.com

1 Analytical and Bioanalytical Chemistry, Springer, Tiergartenstrasse 17, 69121 Heidelberg, Germany
Professor Bernhard Lendl is the recipient of the 2015 Robert Kellner Lecture Award. His lecture, entitled "Mid-IR quantum cascade lasers: a new enabling technology for analytical sciences," was presented at the 18th European Conference on Analytical Chemistry (Euroanalysis 2015), held in Bordeaux, France, from September 6 to 10, 2015.

The Robert Kellner Lecture, sponsored by Springer-Verlag, recognizes a European individual who has made substantial contributions in the last five years to the advancement of analytical chemistry research or education. The award was established by the Division of Analytical Chemistry of the European Association of Chemical and Molecular Sciences in memory of the efforts and achievements of Prof. Robert Kellner, Vienna University of Technology, in particular, towards the consolidation of analytical chemistry in Europe.

\section{Who is Bernhard Lendl?}

Bernhard Lendl has talked about his research, his motivations, and himself to Nicola Oberbeckmann-Winter.

\section{What is the focus of your award-winning research and what was/is your motivation?}

Analytical chemistry is as a highly interdisciplinary scientific discipline, which - among other things - strives to improve the way reliable chemical information can be obtained from samples of different kinds. An important motivation for my research is to make use of recent technological developments and know-how from neighboring disciplines like solid-state electronics and physics. By 
introducing and establishing these advances in the field of analytical chemistry, I aim to elaborate new measurement concepts and test them on real-life samples. Because the major focus of my research concerns midIR spectroscopy, I am particularly interested in new light sources, notably lasers that cover the mid-IR spectral range. Among these, quantum cascade lasers (QCLs) are highly attractive due to their broad spectral coverage, small size, high output power, and room-temperature operation. I feel certain that this novel type of light source will fundamentally change the analytical problem-solving capabilities of mid-IR spectroscopy. For this reason, I would like to contribute to this progress by developing and testing new measurement concepts for gases, liquids, and solids (imaging) at TU Wien, which take advantage of the unique properties of QCLs. If promising new technology emerges, I try to transfer this know-how to our spinoff company QuantaRed Technologies for further exploitation.

\section{What is the trickiest problem you have had to overcome in that research? How did you solve it?}

When we started working with QCLs, merely research prototypes of these lasers were available. Our friends and colleagues from solid-state electronics, who worked on advancing the core technology of these new light sources, clearly had a different focus than we analytical chemists. In the beginning, it was difficult to find a common language and a common ground for joint activities. Furthermore, neither dedicated electronics nor data acquisition systems were available in my laboratory; thus it was challenging to set up the first, meaningful experiments. I could solve these initial problems by building an interdisciplinary research group with members trained in chemistry, physics, solid-state electronics, and biology, which I tried to embed in an international cooperation network.

\section{From where did you start and how does your current work relate to/differ from your scientific roots?}

My scientific roots can be traced to my diploma thesis, which I performed in the laboratory of Prof. Miguel Valcárcel (University of Córdoba, Spain) in the field of flow injection analysis, and to my $\mathrm{PhD}$ thesis under the supervision of Prof. Robert Kellner (Technische Universität Wien, Austria), where I started to work with FTIR spectroscopy. During this period, I focused on working with standard instrumentation inside a classical analytical laboratory. These days, we try to develop new measurement technologies and corresponding instrumentation, which are (often) designed to be used outside an analytical laboratory, e.g., in an industrial setting.

\section{How would you explain your current research to a child?}

Playing with something like LEGO. Assembling and disassembling toys which we build to learn about our nature.

\section{Where do you consider your field is heading?}

Vibrational spectroscopy allows molecular-specific and destruction-free analysis of samples of different kinds by studying their interaction with electromagnetic radiation. Whereas a broad variety of diverse measurement concepts can be envisioned (ranging from stand-off detection to nearfield imaging and from measurement of gases to solids), a specific strength of these techniques is that portable instruments can be designed. I see one trend in that field towards implementation of IR and Raman-based measurement techniques for problem solving in targeted applications that are not necessarily incorporated in a traditional laboratory infrastructure. No doubt there is a great future for IR and Raman techniques in process analytical chemistry, environmental monitoring as well as point-of-care diagnostics. A second trend clearly points towards chemical imaging in the far- and the near-field. In this regard and with the objective of a most complete characterization of a given sample, we currently envision and implement the "advanced combination" of vibrational spectroscopies (IR and Raman) with techniques providing complementary elemental (LA-ICP-MS, SEM-EDX, etc.) and/or structural (MALDI, SIMS, etc.) information. With "advanced combination" I refer to a combined chemometric analysis of data received by different techniques but recorded from the same areas or voxels of the sample under investigation.

\section{Which recent discovery might prove most valuable to the field of bioanalytical research or beyond?}

This is very difficult to say. Maybe for bioanalytical research near-field mid-IR imaging, i.e., the combination of atomic force microscopy and mid-IR laser spectroscopy, might have the brightest future.

\section{What motivated you to become a researcher and what do you find most rewarding in your professional life?}

The motivation to become a researcher was nourished mainly by interest in the subject, the motivation to follow the example 
of my grandfather (he was a professor of geography), as well as the very positive examples of teachers and colleagues I had the chance to work with in the very early stages of my career. As most rewarding I see the independence to define my own field of research, working with motivated young people, enjoying an international network of colleagues and friends, and being able to travel the world.

\section{What is your advice for young analytical scientists today?}

Try to think out of the box and do not be afraid to make mistakes.
From your personal point of view, which $\mathrm{ABC}$ feature do you like (best) and why?

I like the topical collections very much. Furthermore, I enjoy the fact that the Austrian Society of Analytical Chemistry is co-owner of $\mathrm{ABC}$.

And last but not least, what do you enjoy most when you have time to spare?

Many things, mostly I look for quiet moments in nature, spending time with my family, cooking and travelling. 